\title{
The first description of a singular case of synchronous chronic myelomonocytic leukemia and diffuse large b-cell lymphoma
}

\author{
Alessandra Romano $^{1}$ (D) | Michele Giusti ${ }^{2,3}$ | MaryAnn Di Giorgio ${ }^{4}$ | \\ Giovanni Lumera $^{2,3}$ | Nunziatina Laura Parrinello ${ }^{4}$ | Sebastiano Cosentino ${ }^{5}$ | \\ Massimo Ippolito $^{5}$ | Loredana Villari $^{6}$ | Giuseppe Alberto Palumbo $^{7}$ | \\ Francesco Di Raimondo $^{1,4_{*}}$ | Salvatore Santo Signorelli ${ }^{2,3 *}$ \\ ${ }^{1}$ Dipartimento di Chirurgia e Specialità Medico Chirurgiche, Sezione di Ematologia, Università degli Studi di Catania, Catania, Italy \\ ${ }^{2}$ Department of Clinical and Experimental Medicine, Università degli Studi di Catania, Catania, Italy \\ ${ }^{3}$ UO Medicina Generale, AOU Policlinico di Catania, Presidio Rodolico, Catania, Italy \\ ${ }^{4}$ UOC Ematologia, AOU Policlinico di Catania, Presidio Rodolico, Catania, Italy \\ ${ }^{5}$ Dipartimento Tecnologie Avanzate, UOC Medicina Nucleare - Centro PET, AOE, “Cannizzaro” Catania, Catania, Italy \\ ${ }^{6}$ UO Anatomia Patologica, AOU Policlinico di Catania, Presidio San Marco, Catania, Italy \\ ${ }^{7}$ Dipartimento di Scienze Mediche, Chirurgiche e Tecnologie Avanzate "G.F. Ingrassia", Sezione di Ematologia, Università degli Studi di Catania, Catania, \\ Italy
}

\author{
Correspondence \\ Alessandra Romano, Dipartimento di \\ Chirurgia e Specialità Medico Chirurgiche, \\ Sezione di Ematologia, Università degli \\ Studi di Catania, Via Santa Sofia 78, \\ PAD8DII, 95123 Catania. \\ Email: alessandra.romano@unict.it; \\ sandrina.romano@gmail.com
}

Funding information

There is no funding to declare.

\begin{abstract}
In CMML, neoplastic monocytes can be distinguished based on their immunophenotype. Supportive care myeloid growth factors in concomitant extranodal non-Hodgkin Lymphoma are safe.
\end{abstract}

K E Y W O R D S

CMML, filgrastim, lymphoma, monocytes, R-CHOP, supportive care

\section{1 | INTRODUCTION}

Chronic myelomonocitic leukemia (CMML) is a myeloid clonal hematopoietic malignancy, characterized by a persistent increase of absolute monocyte count (AMC $\left.>=1+10^{\wedge} 9 / \mathrm{L}\right)$, accounting for more than $10 \%$ of the white blood cells, circulating in peripheral blood. ${ }^{1}$

According to WHO 2016 classification, CMML is diagnosed when monocytosis persists for at least 3 months and other causes of monocytosis have been excluded; alternatively, a flow cytometry assay excluding reactive monocytosis with at least $94 \%$ of classical $\mathrm{CD} 14^{++} \mathrm{CD} 16^{-}$monocytes circulating in peripheral blood can be conclusive for the diagnosis. ${ }^{2-4}$

CMML is a rare disease, affecting preferentially elderly male patients, but associated with a more favorable course in young patients. ${ }^{1,5}$

As recently described, CMML raises from the combination of hypersensitivity of myeloid precursors to granulocyte-macrophage colony-stimulating factor, myeloid cells dysplasia, and ineffective hematopoiesis. ${ }^{3,6,7}$ Recent advances in molecular profiling lighted the multistep dynamics of CMML pathogenesis, which can be anticipated by oligomonocytic CMML, in

*equally contributed as last authors.

This is an open access article under the terms of the Creative Commons Attribution License, which permits use, distribution and reproduction in any medium, provided the original work is properly cited.

(C) 2021 The Authors. Clinical Case Reports published by John Wiley \& Sons Ltd. 
which AMC does not reach a diagnostic level but all other criteria for CMML are fulfilled, or other clonal and nonclonal conditions, including idiopathic monocytosis (IMUS) and clonal monocytosis of unknown significance (CMUS). In some patients, CMML is anticipated by a diagnosis of clonal cytopenia of unknown significance (CCUS), clonal hematopoiesis of indeterminate potential (CHIP), and idiopathic cytopenia of undetermined significance (ICUS). ${ }^{5}$

To make the picture more complex, in a few patients, CMML is associated with other hematological disease, including lymphoproliferative diseases such as monoclonal gammopathy if uncertain significance (MGUS), monoclonal B-cell lymphocytosis (MBL), multiple myeloma (MM), or non-Hodgkin T- and B-cell lymphomas. ${ }^{1}$ In most cases, the diagnosis of lymphoid neoplasm is detected first and CMML is considered a post-treatment disease, but there is lack of knowledge due to the anecdotal nature of these observations.

Herein, we report the first case described in literature about the synchronous diagnosis of large B-cell lymphoma (DLBCL) and CMML.

\section{1 | Case presentation}

A 68-year-old Caucasian man was referred by the emergency room for anemia, secondary to blood loss due to several episodes of hematemesis and melena. He had a history of not compensated mellitus diabetes type II, diverticulitis, essential hypertension and benign prostatic hyperplasia (BPH) and complained of chronic inflammatory pain for ureteral stenosis, weight loss (3 $\mathrm{kg}$ in the last month) and fever, without nausea or abdominal pain.

On physical examination, his vital signs were normal, and no palpable lympho-adenopathy or splenomegaly. The review of his medical records from the previous two years showed a progressive increase in leucocytosis, including monocytosis, which reached a peak of 5,040 absolute monocyte cells $(\mathrm{AMC}) / \mathrm{mmc}$, in absence of blast at the evaluation of the peripheral blood smear.

Blood count examination showed severe anemia $(\mathrm{Hb}$ $6.4 \mathrm{~g} / \mathrm{dL}$ ) that required repeated blood transfusions, thrombocytopenia (platelet count at baseline $64,000 / \mathrm{mmc}$ ), moderate leucocytosis (white blood cells, WBC, 13,900/mmc), with absolute monocytosis (AMC 3,300/mmc). Laboratory investigations showed elevated levels of lactate dehydrogenase, C-reactive protein, and beta 2 microglobulin. None of cancer markers neither acute infections tests were found as positive.

\subsection{1 | Second level examinations: methods and results}

The esophagus-gastroscopic examination (EGE) $)^{8,9}$ demonstrated a vegetative, ulcerated mass in the stomach antrum and body, worsened a month after with progressive extension and thickening of ulcerated areas.

Histological examination (HE) of the gastric biopsy showed diffuse infiltration of the lamina propria, with replacement in most glands by large lymphoid cells with vesicular nuclei (Figure 1A). The immune histochemical profile was positive for LCA, CD20, PAX5, Bcl-6, and Bcl2 neoplastic cells (Figure 1B, C), associated with high proliferation rate (90\% ki67 index), and negative for CD3, CD5, CD10, Cyclin D1, and CD23 (Figure $1 \mathrm{~A}-\mathrm{D})$, in absence of translocations involving MYC, BCL-2, and BCL- 6 , ${ }^{10}$ thus conclusive for the diagnosis of diffuse large B-cell lymphoma (DLBCL).

Computed tomography (CT) imaging of the chest and abdomen showed a thickening of stomach antrum and body, associated with enlarged lymph nodes (max diameter $18 \mathrm{~mm}$ ) in the perigastric adipose tissue, and two suspect lesions in the left adrenal gland, due to increased enhancement after injection of contrast dye in absence of hepatosplenomegaly (Figure 1E).

18-F-fluoro-2-deoxyglucose (FDG) positron emission imaging showed increased uptake at the level of stomach antrum (standardized uptake value, SUVmax = 34) and skeleton as by bone marrow activation, without involvement of left adrenal gland. The lymphoma staging was completed by the bone marrow examination, which excluded the presence of lymphoproliferative infiltrate, showing dysgranulopoiesis, associated with an increase in the immature granulocyte and monocyte compartment (MPO+, CD33+, CD14+, CD68+) and fibrosis grade 1 (Figure 2A), conclusive for chronic myelomonocytic leukemia (CMML).

As CMML is invariably associated with monocytosis, all causes of reactive monocytosis should be investigated before of a conclusive diagnosis. ${ }^{4}$ Based on the first report of Dr Seligmoglu-Buet and colleagues, ${ }^{3}$ the presence of $>94 \%$ of $\mathrm{CD} 14^{+} \mathrm{CD} 16^{-}$classical monocytes (MO1s) in the peripheral blood is a strong and better predictor of CMML than the $1 \times 10^{9} / \mathrm{L}$ cutoff currently used by the WHO. Also in our case, the immune phenotype of monocytes circulating in peripheral blood was helpful, showing increased absolute numbers of $\mathrm{CD} 14^{+} \mathrm{CD} 16^{-}$classical MO1s and inflammatory $\mathrm{CD} 14^{+} \mathrm{CD} 16^{+} \mathrm{MO} 2 \mathrm{~s}$, associated with lack of $\mathrm{CD} 14^{\text {low }} \mathrm{CD} 16^{+} \mathrm{MO} 3 \mathrm{~s}$ (Figure $2 \mathrm{E}$ ), confirmed at the bone marrow level.

Conventional cytogenetics showed a normal XY karyotype, and molecular profiling identified the single mutation in ASXL1 gene (exon 12), generally associated with inferior outcome in de novo CMML. ${ }^{11}$ Thus, the patient has a high-risk DLBCL, according to the International Prognostic Index, associated with a synchronous diagnosis of CMML-0, ${ }^{1}$ Intermediate- 1 risk according to CPSS-mol score. ${ }^{11}$

The management and treatment of lymphoid neoplasms presenting with concomitant CMML is challenging, due to the lack of large series, and at the best of our knowledge, no other cases of DLBCL have been described before. Due to the 

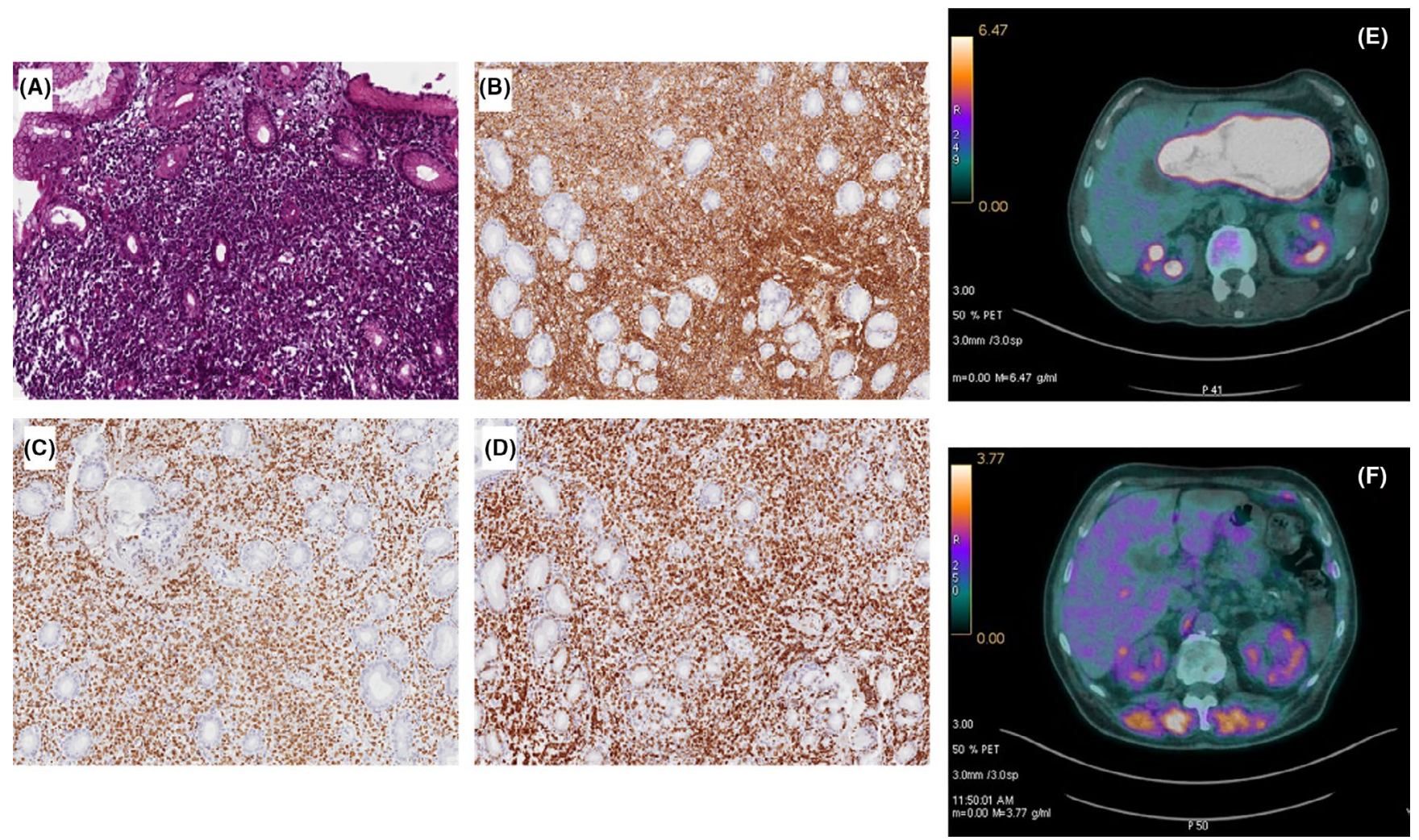

F I G URE 1 Immunohistochemical and imaging features of DLBLC

Photomicrographs of the stomach histology and immunohistochemistry supporting the diagnosis of diffuse large B-cell lymphoma (DLBCL). (A) Hematoxylin and eosin showed a diffuse infiltration of the lamina propria, with replacement of most of the glands by large-sized lymphoid cells with vesicular nuclei replacing the normal gastric structure. Immunohistochemistry showed a positive immunostaining (brown) of the lymphocytes with the B-cell antibody CD20 (B), Bcl-6 (C), and high ki67 index (D). Staging procedures included CT-PET (E) scan, repeated after first four months of treatment (F), compatible with a partial remission.

aggressive clinical course of DLBCL, we planned six cycles of R-CHOP-21 treatment consisting of intra venous injection on day 1 (D1) of Rituximab 375 mg/mq (given $1400 \mathrm{mg}$ subcutaneously from the second cycle onwards), cyclophosphamide $750 \mathrm{mg} / \mathrm{mq}$, vincristine $1.4 \mathrm{mg} / \mathrm{mq}$, and prednisone $100 \mathrm{mg}$ per os on days 1 to 5 . Prednisone was omitted in the first three cycles due to uncontrolled diabetes and risk of perforation, and dosage halfed at cycles 4 and 5. Due to severe constipation, vincristine was given at $1 \mathrm{mg}$ flat dose at cycles 1-2-3 and omitted at cycles 4-5. The last cycle was not given due to dysphagia, abdominal pain, and acute kidney failure due to ureteral stenosis.

Supportive care consisted of prophylaxis with cotrimoxazole $800 \mathrm{mg}$ bid twice a week, acyclovir $800 \mathrm{mg}$ bid, 40,000 UI erythropoietin zeta given once a week, and 30MU filgrastim given at days $+8+9$ of each cycle, and it was sufficient to avoid delays in recycling. Hematological toxicities included neutropenia grade 3 , while anemia and thrombocytopenia improved during the course of treatment (Figure 2). No hematological toxicities were expected and included severe stipsis due to vincristine exposure, dysphagia, and asymptomatic increased of transaminases due to doxorubicine. No active infections were registered.

At the end of the treatment, the gastric lesion was reduced more than 50\%, as documented by CT-PET scans (Figure 1F). After 6 months from the end of treatment, a weak uptake of 18-DG (SUV max 5.3) was still found, but it could be due to lack of resolution of inflammatory status. Bone marrow biopsy showed no residual CMML, and the mutation of ASXL1 was no longer found, despite the persistence of monocytosis, due to increased absolute and percentage numbers of CD14 + CD16- classical MO1s (Figure $2 \mathrm{~F}$ ), and anemia.

At 15 months follow-up, the patient is alive, without gastrointestinal discomfort, maintaining CMML-0 diagnosis, not requiring any additional supportive care.

\section{2 | DISCUSSION}

The synchronous diagnosis of CMML and lymphomas is a quite rare event. Anecdotal cases of association with 

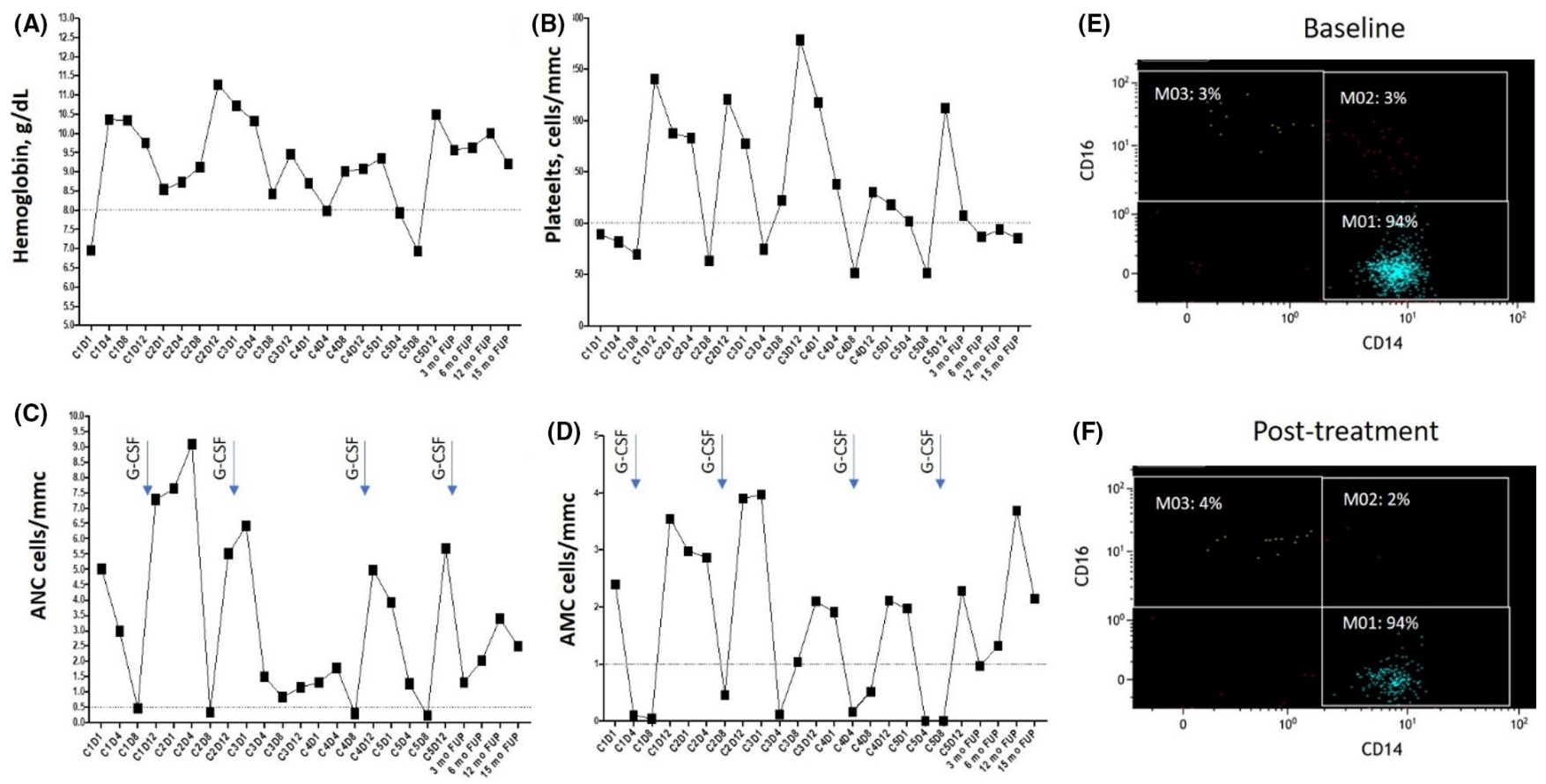

F I G URE 2 Blood counts in peripheral blood during before, during, and after treatment for DLBCL

Hematological parameters in peripheral blood during before, during, and after treatment for DLBCL are reported, referred to hemoglobin, measured in g/dL (A), platelet count/mmc (B), absolute neutrophil count (C), and absolute monocyte count (D). Arrows in (C) and (D) indicate the start of supportive care with G-CSF. Abbreviations: C, cycle, D, day. Monocyte subset repartition based on multiparameter flow cytometry analysis of monocyte (MO) subsets at baseline (E) and after treatment specific for DLBCL (F), showing an increased fraction of classical monocytes (>94\%).

angioimmunoblastic T-cell lymphoma, plasma cell disorders, and marginal B-cell cell lymphoma have been described. ${ }^{12}$ This is the first clinical description of association of CMML with DLBCL.

About $40 \%$ of CMML patients carry mutations in the chromatin remodeling gene ASXL1, ${ }^{13}$ which confers inferior clinical outcome. ${ }^{1}$ In our patient, molecular profiling disclosed a mutation in the ASXL1. Since some mutations, such as those involving ASXL1 and DNMT3a genes, could be associated with clonal hematopoiesis of indeterminate potential and increase the risk of a second blood malignancy, ${ }^{14,15}$ we looked for their involvement in the gastric biopsy. The lack of overlapping mutation profile status between CMML and DLBCL suggested that these two hematological malignancies were not related one each other. After specific chemotherapy for DLBCL, ASXL1 mutation was not detectable anymore in the CMML cells, despite persistence of monocytosis, suggesting that the treatment eradicated a probable founder subclone. ${ }^{16,17}$ The immune phenotype ${ }^{4}$ and the persistence after treatment excluded the expansion of monocytic myeloid derived suppressor cells. ${ }^{18}$

In nontransplantable patients, both diseases require separate treatment plans. ${ }^{1}$ The choose of treatment reflected the need to treat first of all those symptoms related to gastric localized lymphoma. Despite a suspicious lesion in the adrenal gland, we did not perform any prophylaxis against secondary central nervous system disease, a complication that occurs in approximately $5 \%$ of patients with DLBCL, generally within the first year after the diagnosis, due to low platelet count and the increased risk of hematological toxicity. We paid more attention on the administration of GCSF, to avoid the stimulation of the growth of myeloid precursors. In lack of specific guidelines for the supportive care measures in CMML patients, we took advantage of more mature experience in managing myelodysplastic syndromes (MDS) administrating erythropoietin analogs for the treatment of anemia and prophylactic antibiotics and antivirals. ${ }^{5}$ Based on National Cancer Center Network (NCCN) treatment guidelines, the routine use of G-CSF should be avoided in patients with MDS, but at the same time G-CSF injections are recommended as prophylaxis in case of neutropenia with recurring, serious infections, or during infectious episodes. We considered to limit the risk of any infectious disease using an on-demand policy for filgrastim injection, based on the ANC count evaluated at different time points during the cycle. In this way, hematic crasis improved overtime while no infections occurred. 


\section{3 | CONCLUSIONS}

In conclusion, CMML is a rare myeloid disorder that is rarely associated with NHL, as reported in this uncommon presentation. The immune phenotype and the persistence after treatment excluded the expansion of monocytic myeloid derived suppressor cells. It is probable that age relates with the increasing of genetic mutations that can involve also lymphoid maturation lines. A personalized approach is required to personalize the treatment to reduce toxicity and increase the probability of long-term remission, including on-demand filgrastim injection.

\section{ETHICS STATEMENT}

This study was conducted in respect of Declaration of Helsinki.

\section{ACKNOWLEDGMENTS}

Authors thank AIL (Associazione Italiana Leucemia e Linfomi) Sezione di Catania for its unvaluable support.

\section{CONFLICT OF INTEREST}

AR and FDR received honoraria from Amgen, Novartis, and Takeda. NLP and GAP received honoraria from Novartis. All the other authors do not have any conflicts of interest.

\section{AUTHOR CONTRIBUTION}

AR and MG.: described the case and performed literature research. MDG and GL: performed bone marrow aspirate and prepared figures. NLP: performed flow cytometry assays. SC and MI: performed radiological scans. LV: performed immunohistochemistry. GAP: coordinated supportive care. FDR, SS, and AR: designed the study and wrote the manuscript.

\section{DATA AVAILABILITY STATEMENT}

No further data are available.

\section{ORCID}

Alessandra Romano (D) https://orcid.

org/0000-0002-6333-4433

\section{REFERENCES}

1. Valent P, Orazi A, Savona MR, et al. Proposed diagnostic criteria for classical chronic myelomonocytic leukemia (CMML), CMML variants and pre-CMML conditions. Haematologica. 2019;104(10):1935-1949.

2. Vazquez R, Roussel M, Badaoui B, et al. High sensitivity of the Hematoflow ${ }^{\mathrm{TM}}$ solution for chronic myelomonocytic leukemia screening. Cytometry B Clin Cytom. 2018;94(5):658-661.

3. Selimoglu-Buet D, Wagner-Ballon O, Saada V, et al. Characteristic repartition of monocyte subsets as a diagnostic signature of chronic myelomonocytic leukemia. Blood. 2015;125(23):3618-3626.
4. Tarfi S, Harrivel V, Dumezy F, et al. Multicenter validation of the flow measurement of classical monocyte fraction for chronic myelomonocytic leukemia diagnosis. Blood Cancer Journal. 2018;8(11):114.

5. Valent P. Oligo-monocytic CMML and other pre-CMML states: Clinical impact, prognostication and management. Best Pract Res Clin Haematol. 2020;33(2):101137.

6. Itzykson R, Duchmann M, Lucas N, Solary E. CMML: Clinical and molecular aspects. Int J Hematol. 2017;105(6):711-719.

7. Solary E, Itzykson R. How I treat chronic myelomonocytic leukemia. Blood. 2017;130(2):126-136.

8. Vetro C, Chiarenza A, Romano A, et al. Prognostic Assessment and Treatment of Primary Gastric Lymphomas: How Endoscopic Ultrasonography Can Help in Tailoring Patient Management. Clinical Lymphoma Myeloma and Leukemia. 2014;14(3):179-185.

9. Vetro C, Romano A, Amico I, et al. Endoscopic features of gastro-intestinal lymphomas: from diagnosis to follow-up. World $J$ Gastroenterol. 2014;20(36):12993-13005.

10. Tisi MC, Ferrero S, Dogliotti I, et al. MYC Rearranged Aggressive B-Cell Lymphomas: A Report on 100 Patients of the Fondazione Italiana Linfomi (FIL). Hemasphere. 2019;3(6):e305.

11. Elena $\mathrm{C}$, Gallì A, Such E, et al. Integrating clinical features and genetic lesions in the risk assessment of patients with chronic myelomonocytic leukemia. Blood. 2016;128(10):1408-1417.

12. Soriano PK, Stone T, Baqai J, Sana S. A Case of Synchronous Bone Marrow Chronic Myelomonocytic Leukemia (CMML) and Nodal Marginal Zone Lymphoma (NMZL). Am J Case Rep. 2018;19:1135-1139.

13. Abdel-Wahab O, Gao J, Adli M, et al. Deletion of Asxl1 results in myelodysplasia and severe developmental defects in vivo. $J$ Exp Med. 2013;210(12):2641-2659.

14. Jaiswal S, Fontanillas P, Flannick J, et al. Age-related clonal hematopoiesis associated with adverse outcomes. $N$ Engl J Med. 2014;371(26):2488-2498.

15. Genovese G, Kähler AK, Handsaker RE, et al. Clonal hematopoiesis and blood-cancer risk inferred from blood DNA sequence. $N$ Engl J Med. 2014;371(26):2477-2487.

16. Barresi V, Romano A, Musso N, et al. Broad copy neutral-loss of heterozygosity regions and rare recurring copy number abnormalities in normal karyotype-acute myeloid leukemia genomes. Genes Chromosomes Cancer. 2010;49(11):1014-1023.

17. Barresi V, Palumbo GA, Musso N, et al. Clonal selection of $11 \mathrm{q} \mathrm{CN}$ LOH and CBL gene mutation in a serially studied patient during MDS progression to AML. Leuk Res. 2010;34(11):1539-1542.

18. Palumbo GA, Parrinello NL, Giallongo C, et al. Monocytic Myeloid Derived Suppressor Cells in Hematological Malignancies. Int J Mol Sci. 2019;20(21).

How to cite this article: Romano A, Giusti M, Di Giorgio M, et al. The first description of a singular case of synchronous chronic myelomonocytic leukemia and diffuse large b-cell lymphoma. Clin Case Rep. 2021;9:e03817. https://doi.org/10.1002/ ccr3.3817 